\title{
Rekabetçi Evrimsel Algoritmalar ile Yuvarlak Papyon Anten Tasarımı
}

\section{Rounded Bowtie Antenna Design with Competitive Evolutionary Algorithms}

\author{
Ahmet Uluslu ${ }^{*}$ \\ ${ }^{1}$ İstanbul Üniversitesi-Cerrahpaşa, Elektronik ve Otomasyon Bölümü, İstanbul, Orcid No: 0000-0002-5580-1687
}

\begin{tabular}{|c|c|c|}
\hline \multicolumn{2}{|c|}{ MAKALE BİLGİLERİ } & ÖZET \\
\hline \multicolumn{2}{|c|}{ Makale geçmişi: } & $\begin{array}{l}\text { Bu çalışmada kablosuz yerel alan ağları (WLAN) uygulamaları için } 5 \mathrm{GHz} \text { bandındaki yuvarlak papyon antenin } \\
\text { tasarım parametrelerinin seçimi rekabetçi evrimsel algoritmalar ile çok boyutlu ve çok amaçlı bir tasarım }\end{array}$ \\
\hline Geliș: & 10 Nisan 2021 & optimizasyon problemi olarak sunulmuştur. Bu zorlu tasarım optimizasyonun üstesinden gelebilmek için yeni, hizlı \\
\hline Düzeltme: & 28 Temmuz 2021 & ve güçlü optimizasyon algoritmaları, ateşböceği, diferansiyel evrim ve guguk kuşu arama kullanılarak anten \\
\hline Kabul: & 27 Ağustos 2021 & ptimizasyon işlemi rekabetçi bir biçimde karşılaştırılmıştır. Önerilen anten tasarımı ateşböceği algoritması ile \\
\hline \multicolumn{2}{|c|}{ Anahtar kelimeler: } & $\begin{array}{l}\text { olarak, önerilen tasarım optimizasyon süreçleri, tüm anten tasarım problemleri için verimli, hızlı ve güvenilir bir } \\
\text { çözümdür. }\end{array}$ \\
\hline
\end{tabular}

ateşböceăi, difer

guguk kuşu arama

\begin{tabular}{l} 
ARTICLE INFO \\
\hline Article history: \\
Received: $\quad 10$ April 2021 \\
Revised: $\quad 28$ July 2021 \\
Accepted: $\quad 27$ August 2021 \\
\hline Keywords: \\
Optimization, WLAN, $5 \mathrm{GHz}$, \\
firefly, differential evolution, \\
cuckoo search
\end{tabular}

\begin{abstract}
In this study, the selection of the design parameters of the round bowtie antenna in the $5 \mathrm{GHz}$ band for wireless local area networks (WLAN) applications is presented as a multi-dimensional and multi-purpose design optimization problem with competitive evolutionary algorithms. To overcome this challenging design optimization, the antenna optimization process was competitively compared using new fast and powerful optimization algorithms, firefly, differential evolution, and cuckoo search. The return loss (S11) value at the 5 $\mathrm{GHz}$ resonance frequency point designed with the proposed antenna design firefly algorithm was found to be $56.31 \mathrm{~dB}$. As a result, the proposed design optimization processes are an efficient, fast and reliable solution for all antenna design problems.
\end{abstract}




\section{Giriş}

Kablosuz yerel alan ağları (WLAN) ve dipol antenler endüstriyel, tıbbi ve ticari uygulamalarda sıklıkla kullanılmaktadır. $\mathrm{Bu}$ WLAN sistemleri için ayrılan spektrumlar 2,4, 5 ve $5,8 \mathrm{GHz}$ olarak belirtilmiştir [1]. Kablosuz sistemler için bildirilen birçok tek bant veya çift bantlı anten tasarımları mevcuttur [1]- [5]. Çok bantlı anten tasarımları ile birlikte [6]-[8], yakınlardaki iletişim sistemlerine müdahale etmekten kaçınmak için, yalnızca istenen frekans bantlarında çalışabilen bir anten tasarımı da gerekli olabilmektedir. Çoğu durumda, anten boyutu büyüktür ve antenin geometrisi karmaşıktır. Bazı durumlarda da anten kompakt olmasına rağmen oldukça yüksek düzeyde çapraz polarizasyon gösterir [8]. Ayrıca daha yüksek geri dönüş kaybına sahip cihazlara olan talebin artışı kablosuz iletişim sistemlerinin gelişiminde önemli bir rol oynadi. Şu anda her alanda bulunan akıllı binalar, bilgi işlem ve sensor ağlar [9]-[10] da dahil olmak üzere birçok yeni uygulamalar için farklı iletişim frekansları, veri hızları ve kapasiteler sunan birçok kablosuz LAN sistemleri mevcuttur. Bu tür sistemler dar-geniş bantlı ve birçok frekans üzerinde çalışabilen ve aynı zamanda taşınabilir cihazlarda imal edilebilecek kadar küçük olan minyatür geniş bant antenleri gerektirir. Dipol antenler düşük profilli, hafif ve ucuzdur. Entegre devrelerle entegrasyonu kolaydır; bu da onları taşınabilir cihazlarda kullanıma uygun hale getirir. Bununla birlikte, geleneksel dipol antenler, nispeten dar bant genişlikleri ile karakterize edilir. Araştırmacılar, dipol antenlerin geri dönüş kaybını ve kazancını iyileştirmek için birçok iyileştirme yöntemi geliştirmiştir [11]-[14]. Bu iyileştirmeler için başlangıç noktası doğru geometrik boyut seçimidir. İşte bu noktada devreye giren optimizasyonun kullanılmas1, geleneksel teknikler kullanılarak tasarımların araştırılmasına izin verir. Geometri içeren uygulamalarda genetik algoritmalar (GA) [12] diğer optimizasyon tekniklerine kıyasla daha gelişmiş özelliklere sahip olduğu görülmektedir [13]-[14]. Dipol anten tasarımında, önceden belirlenmiş şekildeki antenin genetik algoritma kullanarak optimum boyutlarının tespiti tekniklerine odaklanmaktadır. Buradaki yaklaşım, antenin boyutunu, karmaşıklığını veya maliyetini artırmadan dipol antenleri tasarlamak için özellikle uygun ve güçlü bir sınırlandırılmamış GA uygulamasını uygular.

Bir sonraki bölümde, kullanılacak olan anten tasarımı sunulmaktadır. Akabinde, bu çalışmada kullanılacak olan algoritmalar akış diyagramları ile anlatılacaktır. Amaç ve maliyet fonksiyonlarının belirlenmesinden sonra yapılan çalışma kısmı anlatılacaktır. Sonuçlarımız, tüm antenlerde kullanılabilecek bazı önerilerle birlikte son bölümde özetlenmiştir.

\section{Anten Tasarımı}

\section{Yuvarlak Papyon Anten}

Bir yuvarlak papyon anteni, iki konik çift kutuplu antenlerin düzlemsel bir şeklidir. Yuvarlak papyon antenler, hafif ağırlıklı, düşük maliyetli, düşük profilli ve simetrik radyasyon modeli gibi birçok avantaja sahiptir; bu da onları iletişim sistemleri, kablosuz yerel ağları (WLAN) vb. uygulamalar için yaygın olarak kullanılan anten modellerinden biri yapar [16]-[21]. Bununla birlikte, tasarımın performans ölçüleri büyük ölçüde geometrik tasarım değerlerine bağlıdır. Bu nedenle, yuvarlak papyon antenlerin tasarımı, çok amaçlı, çok boyutlu bir tasarım optimizasyon problemi olarak düşünülebilir. Anten tasarımının üç farklı rekabetçi algoritma ile optimizasyonu için bu çalışmada, üçgen bir dipol yuvarlak papyon anteni dikkate alınmıştır. Anten tasarımının şematiği ve tasarım parametreleri Şekil 1 ve Tablo 1'de verilmiştir.

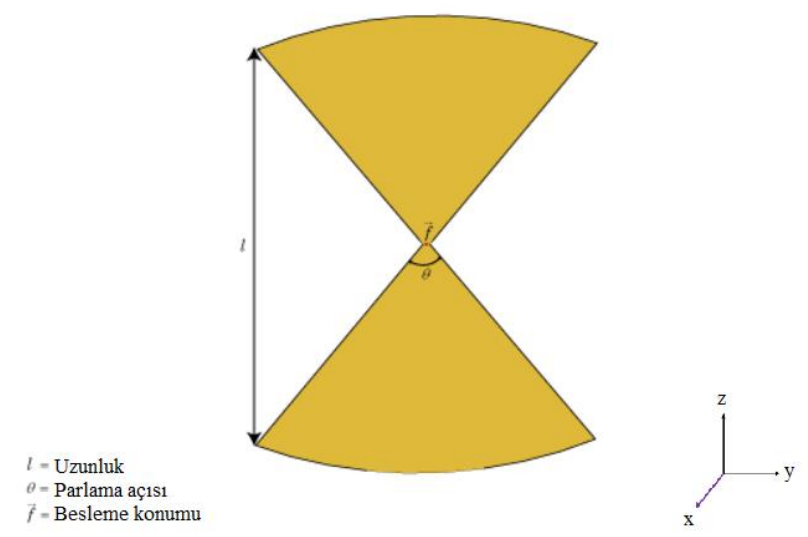

Şekil 1. Yuvarlak papyon anten

Figure 1. Rounded bowtie antenna 
Tablo 1. Anten tasarımı için parametre aralıkları

Table 1. Ranges of parameters for antenna design

\begin{tabular}{cc}
\hline Uzunluk (metre) & Genişleme açısı (derece) \\
\hline $0.001-0.040$ & $5-90$ \\
\hline
\end{tabular}

Yuvarlak papyon anteninin tasarım optimizasyonu Tablo 1'de verilen optimizasyon değişkenleri kullanılarak rekabetçi evrimsel algoritmalar kullanılarak yapılmıştır.

\section{Evrimsel Algoritmalar}

$\mathrm{Bu}$ çalışmada, bir dipol antenin optimizasyon sürecinde ateşböceği (FF), diferansiyel evrim (DE), guguk kuşu (CS) olmak üzere üç rekabetçi evrimsel algoritma kullanılmış ve bunların performans değerlendirmeleri doğruluk ve hesaplama verimliliği açısından karşılaştırılmıştır. Ancak, bu algoritmaların ortak özellikleri basit, birkaç kontrol parametresi ile verimli olmaları ve ayrıca bu algoritmaların GA, PSO gibi standart evrimsel algoritmalar üzerindeki üstünlüğünün hesaplama ve mühendislik uygulamalarında gösterilmesidir [22]-[30]. Aşağıdaki alt bölümlerde ateşböceği (FF), diferansiyel evrim (DE), guguk kuşu arama (CS) optimizasyon algoritmaları k1saca verilmektedir.

\section{Ateșböceği}

Ateşböceği (FF) algoritması, Yang [22]-[23] tarafından geliştirilen, doğadaki ateş böceklerinin parlama davranışından esinlenen evrimsel bir optimizasyon algoritmasıdır. Ateşböceği algoritmasında iki önemli özellik vardır: Parlaklık (1şık yoğunluğu) I ve çekicilik $\beta$. Ateşböceği algoritmasında üç standart kural vardır: (i) Bir ateş böceğinin parlaklığı, belirli bir problemin $f_{(x)}$ amaç fonksiyonunun değeriyle belirlenir. Bir küçültme problemi için parlaklık, amaç fonksiyonu $\mathrm{I}_{(\mathrm{x})} \infty 1 / \mathrm{f}_{(\mathrm{x})}$ değeriyle ters orantılı olabilir. (ii) Bir ateş böceğinin çekicilik derecesi parlaklığı ile orantılıdır. Sabit 1şık soğurma katsayısına sahip belirli bir ortamın üssel soğurması nedeniyle mesafe arttığında parlaklık azalacaktır:

$I=I_{0} e^{-\gamma r_{i j}^{2}}$
Burada parlaklık (1şık yoğunluğu) I, ateşböceğinin maksimum parlaklı̆̆ $\mathrm{I}_{0}$ ve 1 şık soğurma katsayısı $\gamma$ ile gösterilmiştir.

Herhangi iki ateşböceği arasındaki mesafe:

$r_{i j}=\left\|\vec{x}_{i}-\vec{x}_{j}\right\|=\sqrt{\sum_{k=1}^{D}\left(x_{i k}-x_{j k}\right)^{2}}$

Burada D, optimizasyon değişken vektörünün boyutudur; $\mathrm{I}_{0}$, ateş böceğinin maksimum parlaklığıdır. Bir ateş böceğinin hareket ettiği mesafe çekiciliğe göre belirlenir. Ateş böceğinin çekiciliği $\beta$ şu şekilde tanımlanır:

$\beta=\beta_{0} e^{-\gamma r_{i j}^{2}}$

$\beta_{0} \mathrm{r}=0$ 'daki çekiciliktir. (iii) Tüm ateşböcekleri üniseks ve cinsiyetlerine bakılmaksızın daha çekici ve daha parlak olanlara doğru hareket edecekler. Belirli bir ateşböceğinden daha parlak veya daha çekici bir ateşböceği yoksa, rastgele hareket edecektir. Böylece bir ateşböceğinin hareketi i daha çekici (daha parlak) başka bir ateşböceğine çekilir, $\mathrm{j}$ tarafından belirlenir.

$\overrightarrow{x_{i}}=\vec{t}=\vec{x}_{i}+\beta\left(\vec{x}_{j}-\vec{x}_{i}^{t}\right)+\alpha_{t} \vec{\varepsilon}_{i}$

$\beta_{0}=1$ ve $\vec{\varepsilon}_{i}^{t}[-1 / 2,1 / 2]$ şeklinde eşit olarak dağıtılmış rastgele sayıların bir vektörü ve $\alpha_{t}$ rasgele dağıtma faktörü olduğunda, genellikle [31]'de olduğu gibi her ardışık yinelemede azaltılır, ancak bu çalışmada rastgele $\alpha_{t} \in[0,1]$.

Algoritma 1: Ateşböceği Algoritmasının Sözde Kodu

Parametreler, PopBoyut, Max_İteration, $\gamma, \mathrm{I}_{0}, \beta$, $\varepsilon_{\text {Req }}$ aşağıdaki gibidir.

(1) PopBoyut: Ateşböceği popülasyonunun boyutu.

(2) Mak_İterasyon: Maksimum yineleme say1s1.

(3) $\gamma$ : Işık soğurma katsayısı.

(4) $\mathrm{I}_{0}$ : Ateşböceğinin maksimum parlaklığı.

(5) $\beta$ : Bir ateş böceğinin çekiciliği.

(6) $\varepsilon_{\text {Req }}$ : İstenen arama hassasiyeti.

Gereksinim: PopBoyut, Mak_Iterasyon, $\gamma, \beta, \varepsilon$ ve maliyet fonksiyonu. 
D boyutlu arama alanı içinde ateşböceklerinin konumlarını rastgele olarak başlatın.

WHILE Iter $<$ Mak_Iter VE $\varepsilon_{\text {Req }}<\varepsilon$

FOR J $=1$ için: PopBoyut

Kullandığım her ateş böceğinin parlaklığını hesaplayın (1-2).

\section{END}

Hedef fonksiyon değerine göre maksimum parlaklık $\mathrm{I}_{0}$ olan ateş böceğini seçin.

FOR $\dot{\mathrm{I}}=1$ için: PopBoyut

(1-2) kullanarak ateşböceği bağıl parlaklığını hesaplayın, ateş böceğinin hareket yönünü göreceli parlaklıkla belirleyin.

Çekiciliği hesaplayın $\beta$ (3)'e göre, ateş böceklerinin uzay konumlarını (4)'e göre güncelleyin.

END

END

\section{Diferansiyel Evrim}

Diferansiyel evrim (DE) algoritması, gerçek değerli sayısal optimizasyon problemlerinin çözümü için geliştirilmiş popülasyon tabanlı evrimsel bir optimizasyon algoritmasıdır [27-30]. Geleneksel benzerlerinden farklı olarak, amaç fonksiyon manzarasını keşfetmek için parametre vektörlerinin farkını kullanan çok etkili bir küresel optimizasyon algoritmasıdır. Diferansiyel evrim algoritması, genetik algoritmanın mutasyon, çaprazlama ve seçim stratejilerini, geçiş sabiti $\mathrm{C}_{\mathrm{r}} \in(0,1)$, mutasyon ölçek faktörü $\mathrm{F} \in(0,2)$ ve popülasyon boyutu olan çok az sayıda kontrol parametresi ile kullanır. $\mathrm{Bu}$ parametrelerin algoritmanın performans1 üzerindeki etkileri iyi incelenmiştir. Daha iyi çözümler oluşturmadaki temel farklılık, genetik algoritmaların çapraz geçişe dayanması, diferansiyel evrimin ise mutasyon işlemine dayanmasıdır. $\mathrm{Bu}$ ana işlem, popülasyondaki rastgele örneklenmiş çözüm çiftlerinin farklılıklarına dayanmaktadır. Algoritma, aramayı arama alanındaki muhtemel bölgelere yönlendirmek için bir arama mekanizması ve seçim işlemi olarak mutasyon işlemini kullanır.
D parametrelerinden oluşan bir optimizasyon görevi, D boyutlu bir vektör ile temsil edilebilir. Diferansiyel evrimde, bașlangıçta rastgele bir popülasyon büyüklüğü (NP) çözüm vektörleri popülasyonu oluşturulur. $\mathrm{Bu}$ popülasyon, mutasyon, geçiş ve seçim operatörleri uygulanarak başarılı bir şekilde geliştirilir.

Algoritma 2: Diferansiyel Evrim Algoritmasının Sözde Kodu

Parametreler, PopBoyut, Mak_İterasyon, F, C $\mathrm{C}_{\mathrm{r}}$, $\varepsilon_{\text {Req }}$ aşağıdaki gibidir.

(1) PopBoyut: Popülasyon boyutu.

(2) Mak_İterasyon: Maksimum yineleme say1s1.

(3) F: Ölçek Faktörü,

(4) $\mathrm{C}_{\mathrm{r}}$ : Çapraz geçiş,

(5) $\varepsilon_{\text {Req }}$ I İstenen arama hassasiyeti.

Gereksinim: PopBoyut, Mak_İterasyon, F, C $\mathrm{r}$, $\varepsilon$ ve maliyet fonksiyonu.

$\mathrm{G}=0$ oluşturma numarasını ayarlayın ve PopBoyut bireylerinin popülasyonunu rastgele başlatın.

WHILE Iter $<$ Mak_İter VE $\varepsilon_{\text {Req }}<\varepsilon$

IF $\quad \dot{I}=1$ için: PopBoyut

Mutasyon:

DE'nin

$\vec{v}_{i, G}=\vec{x}_{r_{1}^{i}, G}+F .\left(\vec{x}_{r_{2}^{i}, G}-\vec{x}_{r_{3}^{i}, G}\right) * \quad$ olarak diferansiyel mutasyon şeması aracılığıyla i'inci hedefe karşıllı gelen bir donör vektörü oluşturun.

Çaprazlama: Aşağıdaki şekilde iki terimli geçiş yoluyla i'nci hedef vektör $\vec{u}_{i, G}=\left[u_{1, i, G}, u_{2, i, G}, u_{3, i, G}, \ldots . u_{D, i, G}\right] \quad$ için bir deneme vektörü $\vec{x}_{i, G}$ oluşturun

FOR $\mathrm{J}=1: \mathrm{D}$

IF $\quad\left(\operatorname{rand}_{i, j}[0,1] \leq C_{r}\right.$ or $\left.j=j_{\text {rand }}\right)$

$u_{j, i, G}=v_{j, i, G}$

ELSE

$u_{j, i, G}=x_{j, i, G}$ 


\section{END}

Seçim: Deneme vektörünü değerlendirin

$$
\begin{aligned}
& \text { IF } f\left(\vec{u}_{i, G}\right) \leq f\left(\vec{x}_{i, G}\right) \\
& \vec{x}_{i, G+1}=\vec{u}_{i, G}
\end{aligned}
$$

\section{ELSE}

$$
\vec{x}_{i, G+1}=\vec{x}_{i, G}
$$

END

\section{END}

\section{END}

*: $\{1,2, \ldots$, PopBoyut $\}$ içinde rastgele seçilir ve

**: $\{1,2,3, \ldots . ., \mathrm{D}\}$ 'de rastgele seçilir

\section{Guguk Kuşu Arama}

Son zamanlarda, guguk kuşu arama (CS) Yang ve Deb [24] tarafından doğadan ilham alan yeni bir popülasyon tabanlı algoritma olarak sunuldu ve küresel yakınsama özelliklerini garanti ettiği kanıtlandı [24-27]. Standart meta-sezgisel GA, özellikle çok amaçlı optimizasyon problemleri için maliyetli olduğu, uygunluk fonksiyonunun tekrar tekrar değerlendirilmesini gerektirmeyen ve makul bir süre içinde bir dizi optimum çözüm sağlayabildiği durumlarda, uygunluk işlevinin tekrarlanan değerlendirmelerine ihtiyaç duyar. Guguk kuşu arama, guguk kuşlarının üreme davranışından ilham alan basit ve hızlı yakınsak bir algoritmadır. Aşağıdaki idealleştirilmiş üç kurala dayanmaktadır: 1) Her guguk kuşu bir seferde bir yumurta (aday çözüm) bırakır ve yumurtasını rastgele seçilen bir yuvaya atar; 2) Yüksek kaliteli yumurtalara sahip en iyi yuvalar (daha iyi çözümler) bir sonraki nesle aktarılacaktır; 3) Mevcut konakçı yuvaların sayısı sabitlenir ve bir guguk kuşunun yumurtladığ yumurta, $p_{a} \in[0,1]$ olasıllığ ile ev sahibi kuş tarafindan keşfedilir. Bu durumda, ev sahibi kuş yumurtayı atabilir veya yuvayı terk edip tamamen yeni bir yuva kurabilir. Kisaca özetlenirse, bir yuvadaki her yumurta bir aday çözümü temsil eder; amaç, yuvalarda pek de iyi olmayan bir çözümü değiştirmek için yeni ve potansiyel olarak daha iyi çözümleri (guguklu yumurtalar) keşfetmektir. $\mathrm{Bu}$ çalışmada performans değerlendirme ve karşılaştırma amacıyla NPE
$(10,100)$ alınmıştır. Bu üç kurala dayanarak, Guguk Kuşu Aramasının (CS) temel adımları, Bileşik Sağ Sol El İletim Hattı (CRLH TL) ultra geniş bant eşleştirme devrelerinin tasarım optimizasyonunda [27] 'deki PSO ve GA ile karşılaştırıldığında başarıyla uygulanmıştır.

Guguk i diyelim ki için yeni çözümler üretirken $x(t+1)$, bir Levy uçuşu gerçekleştirilir.

$$
\begin{aligned}
& \overrightarrow{x_{i}(t+1)}=\overrightarrow{x_{i}}+\vec{\alpha} \oplus L e^{\prime} v y(\lambda), \\
& \vec{\alpha}=\alpha_{0}\left(\vec{x}_{j}^{(t)}-\vec{x}_{i}^{(t)}\right)
\end{aligned}
$$

Eşitlik'e alternatif olarak. (6), Eşitlikteki vektör. (5), ilgili problemin ölçekleriyle ilişkili olması gereken adım boyutu olan bir skaler ile ikame edilebilir. Çoğu durumda kullanabiliriz. Ürün $\oplus$, giriş açısından çarpımlar anlamına gelir. Lévy uçuşu ile rastgele yürüyüş, adım uzunluğu vergi dağılımına göre çizildiği için arama alanını keşfetmede daha etkilidir [26]. Kullanılan guguk kuşu arama algoritmasının sözde kodu aşağıdaki şekilde verilmiştir:

\section{Algoritma 3: Guguk Kuşu Arama}

Algoritmasının Sözde Kodu [27]

Parametreler, yuvaBoyutu (N), Mak_İterasyon, $\mathrm{p}_{\mathrm{a}}, \varepsilon_{\text {Req }}$ aşağıdaki gibidir.

(1) yuvaBoyutu (N): Konak yuvalarının sayıs1.

(2) Mak_İterasyon: Maksimum yineleme sayis1.

(3) $p_{a}$ : Yumurtlayan yumurtanın konukçu tarafından keşif olasılığı,

(4) $\varepsilon_{\text {Req }}:$ İstenen arama hassasiyeti.

Gereksinim: yuvaBoyutu, Mak_İterasyon, $\varepsilon$ ve maliyet işlevi.

$\mathrm{N}$ konak yuvasının popülasyonunu başlatın.

WHILE Iter $<$ Max_İter $\mathrm{VE} \varepsilon_{\text {Req }}<\varepsilon$

Levy uçuşlarıyla Rastgele guguk alın,

Maliyetini / fitness $\mathrm{F}_{\mathrm{i}}$ değerini değerlendirin, yuvaBoyutu $(\mathrm{N})$ arasından bir yuva seçin,

*EGER $\quad \mathrm{F}_{\mathrm{i}}<\mathrm{F}_{\mathrm{j}}$ 
J'yi yeni çözümle değiştirin

\section{END}

Daha kötü yuvaların bir kısmını $\left(\mathrm{p}_{\mathrm{a}}\right)$ terk edin, Toplama uçuşları aracılığıyla yeni konumlarda yenilerini inşa edin.

En iyi çözümleri veya yuvaları kaliteli çözümlerle tutmak,

Çözümleri sıralayın ve mevcut en iyi olanı bulun,

\section{END}

\section{*: j, rastgele seçilen yuvayı patlatır.}

\section{Amaç ve Maliyet Fonksiyonları}

Anten ölçüm fonksiyonları arasında, geri dönüş kaybı $\left(\mathrm{S}_{11}\right)$ ve 90 derecede yönlülük kazanc1 referans noktası olarak seçilmiştir. Moment yöntemi (MoM) tekniği, karmaşık integral denklemlerinin doğrusal denklem sistemine indirgenip, ağırlaştırılmış artıklar (weighted residuals) kullanarak, çözülmesine dayanır. Bütün ağırlaştırılmış artıklar teknikleri bir ya da birkaç parametreye bağlı deneme çözümleri üretilmesine dayanır [32]. Bu tekniğe dayalı olarak aşağıdaki amaç fonksiyonu tanımlanmıştır.

Amaç fonksiyonu:

$$
\begin{aligned}
& \mathrm{AF} 1=\min \left\{\mathrm{e}^{(-\mathrm{yönlendiricilik/wc1)}}\right\} \\
& \mathrm{AF} 2=\min \left\{\mathrm{e}^{(\text {geriDonusKayb1/wc2)}}\right\}
\end{aligned}
$$

burada yönlendiricilik $\geq 0$ ve geriDonusKaybı $<=$ 0 referans noktaları olarak verilmiştir. Amaç işlevi özetlenir ve aşağıdaki gibi tek bir maliyet işlevi oluşturmak için kullanılır:

$$
\text { maliyet }=\mathrm{AF} 1+\mathrm{AF} 2
$$

$5 \mathrm{GHz}$ frekansında önceden tanımlanmış performans parametreleri için analiz yapılması gerektiğinden, optimizasyon sürecinde kullanılacak amaç fonksiyonları (7)-(8) seçilmiştir. Belirlenen hedef fonksiyonları ile 10 farklı koşu üzerinden alınan minimum ortalama maliyet (9) ile sonuç belirlenmeye çalışılmıştır.

$\mathrm{Bu}$ optimizasyon sürecinde karar değişkenleri sırasıyla anten uzunluğu (metre) ve genişleme açısıdır (derece). İhtiyaçların önemi farklı olduğundan farklı ağırlık katsayıları (wc1, wc2) için denemeler yapılmıştır.

\section{Çalışma Kısmı}

Genetik algoritmaların birçoğu aramaya bir nokta yerine bir dizi noktadan başlar. Bu sebepten ötürü algoritma parametrelerinin en optimumunu seçmek çok önemlidir. Kullanılan algoritmalardan ateşböceği algoritması için 1 şık soğurma katsayısı $(\gamma)=0,3$, bir ateş böceğinin çekiciliği $(\beta)=0,5$ ve istenen arama hassasiyeti $\left(\varepsilon_{\text {Req }}\right)=0,5$ olarak alınmıştır. Diferansiyel evrim algoritması için ise ölçek faktörü $(F)=0,5$, çapraz geçiş $\left(\mathrm{C}_{\mathrm{r}}\right)=0,5$ ve arama hassasiyeti $\left(\varepsilon_{\text {Req }}\right)=0,5$ olarak alınmıştır. Guguk kuşu algoritması için ise yumurtlayan yumurtanın konukçu tarafindan keşif olasılığı $\left(\mathrm{p}_{\mathrm{a}}\right)=0,5$ ve istenen arama hassasiyeti $\left(\varepsilon_{\text {Req }}\right)=0,5$ olarak alınmıştır. Tüm algoritmalar için maksimum iterasyon $=30$ ve popülasyon $(\mathrm{N})=30,50$ ve 80 olarak seçilmiştir. Ayrıca optimizasyon algoritmaları rastgele başlatılmış koşullara ve sürece dayalı olduğundan, sabit maksimum iterasyon ve farklı popülasyon parametreleri için diğer optimizasyon problemlerindeki gibi algoritma kodu 10 kere koşturulmuştur.

\section{Optimizasyon için Optimal Parametre Seti Seçimi}

Çalışmanın bu kısmında 3 algoritmanın (ateşböceği, diferansiyel evrim ve guguk kuşu arama) popülasyon $=30,50$ ve 80 için 10 farklı koşusundan seçilen en iyi performansın tekrarı ile tipik maliyet ve fonksiyon hesaplama sayıs1 (FEN) varyasyonları sırası ile Şekil 2, Șekil 3 ve Şekil 4'te gösterilmiştir. Ek olarak, Şekil 2, Şekil 3 ve Şekil 4'teki maliyet ve FEN varyasyonları sayısal tablo olarak Tablo 2, Tablo 3 ve Tablo 4'de verilmiștir. Grafik ve tablolara göre diferansiyel evrim ve guguk kuşu arama algoritmaları için popülasyon $(\mathrm{N})=50$ ve ateşböceği algoritması için ise popülasyon $(\mathrm{N})=$ 80 olarak seçilmiștir. Çalışmanın bir sonraki bölümünde, seçilen optimum parametrelerle devam edilecektir. 


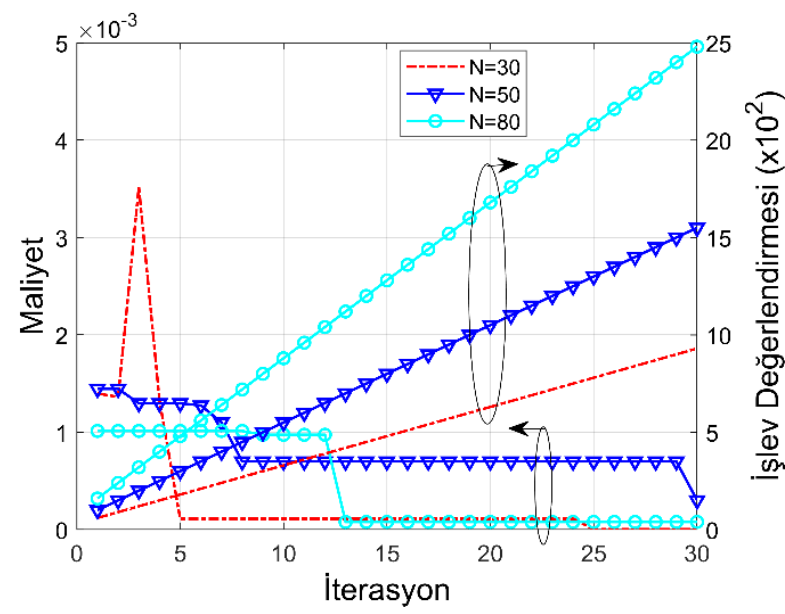

Şekil 2. Ateşböceği algoritması için popülasyona göre 10 farkl koşudan seçilen optimizasyonun en iyi performansinin yinelemeli tipik maliyet ve FEN varyasyonlart

Figure 2. Typical cost and FEN variations with iteration of the best performance of optimization selected from 10 different runs by population for the firefly algorithm

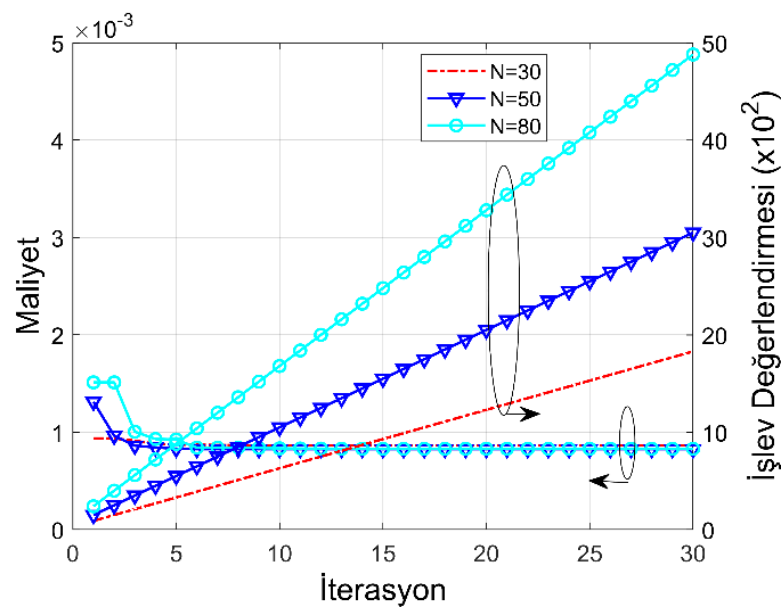

Şekil 3. Diferansiyel evrim algoritması için popülasyona göre 10 farklı koşudan seçilen optimizasyonun en iyi performansinin yinelemeli tipik maliyet ve FEN varyasyonlart

Figure 3. Typical cost and FEN variations with iteration of the best performance of optimization selected from 10 different runs by population for the differential evolution algorithm

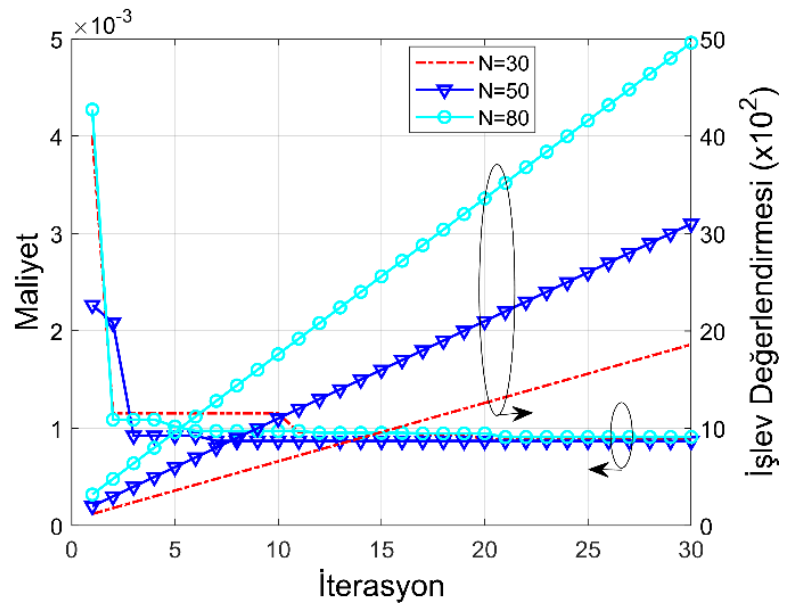

Şekil 4. Guguk kuşu arama algoritması için popülasyona göre 10 farklı koşudan seçilen optimizasyonun en iyi performansinin yinelemeli tipik maliyet ve FEN varyasyonlarl

Figure 4. Typical cost and FEN variations with iteration of the best performance of optimization selected from 10 different runs by population for the cuckoo search algorithm

Tablo 2. Ateşböceği algoritması için popülasyona göre optimizasyonun performans değerlendirmeleri

Table 2. Performance evaluations of optimization by population for the firefly algorithm

\begin{tabular}{ccccc}
\hline Popülasyon & & Minimum & Maksimum & Ortalama \\
\hline \multirow{2}{*}{30} & Maliyet & $3,95 \mathrm{E}-06$ & 0,001397459 & $2,56 \mathrm{E}-04$ \\
& FEN & 780 & 60 & 930 \\
\multirow{2}{*}{50} & Maliyet & 0,000301329 & $1,45 \mathrm{E}-03$ & $8,28 \mathrm{E}-04$ \\
& FEN & 1550 & 100 & 1550 \\
\multirow{2}{*}{80} & Maliyet & $7,89304 \mathrm{E}-05$ & $1,01 \mathrm{E}-03$ & $4,47 \mathrm{E}-04$ \\
& FEN & 1120 & 160 & 2480 \\
\hline
\end{tabular}


Tablo 3. Diferansiyel evrim algoritmast için popülasyona göre optimizasyonun performans değerlendirmeleri

Table 3. Performance evaluations of optimization by population for the differential evolution algorithm

\begin{tabular}{ccccc}
\hline Popülasyon & & Minimum & Maksimum & Ortalama \\
\hline \multirow{2}{*}{30} & Maliyet & $8,64 \mathrm{E}-04$ & 0,000936918 & $8,72 \mathrm{E}-04$ \\
& FEN & 1710 & 90 & 1830 \\
50 & Maliyet & 0,000826929 & $1,31 \mathrm{E}-03$ & $8,50 \mathrm{E}-04$ \\
& FEN & 2350 & 150 & 3050 \\
& Maliyet & 0,000830396 & $1,51 \mathrm{E}-03$ & $8,90 \mathrm{E}-04$ \\
& FEN & 4880 & 240 & 4880 \\
\hline
\end{tabular}

Tablo 4. Guguk kuşu arama algoritması için popülasyona göre optimizasyonun performans değerlendirmeleri

Table 4. Performance evaluations of optimization by population for the cuckoo search algorithm

\begin{tabular}{ccccc}
\hline Popülasyon & & Minimum & Maksimum & Ortalama \\
\hline \multirow{2}{*}{30} & Maliyet & $8,84 \mathrm{E}-04$ & 0,004004882 & $1,09 \mathrm{E}-03$ \\
& FEN & 1260 & 120 & 1860 \\
\multirow{2}{*}{50} & Maliyet & 0,000868378 & $2,27 \mathrm{E}-03$ & $9,64 \mathrm{E}-04$ \\
& FEN & 2900 & 200 & 3100 \\
\multirow{2}{*}{80} & Maliyet & 0,000909947 & $4,27 \mathrm{E}-03$ & $1,07 \mathrm{E}-03$ \\
& FEN & 3520 & 320 & 4960 \\
\hline
\end{tabular}

\section{Maliyet için Ağırlık Katsayısı seçimi}

Seçilen algoritmaların çalışma prensiplerinde en düşük maliyet değerine sahip sonuç bulunmaya çalışıldığından maliyet fonksiyonu içerisindeki ağırlık katsayı parametrelerinin seçimi büyük önem taşımaktadır. Belirlenen maliyet fonksiyonunda 90 derecede yönlendiricilik kazancı parametresinin ağırlık katsayıs1-1 (wc1) ve geri dönüş kayb1 $\left(\mathrm{S}_{11}\right)$ parametresinin ağırlık katsayısı-2 (wc2) olarak görülüyor. Şekil 5, Şekil 6 ve Şekil 7'de ağırlık katsayıs1-1 (wc1) ve ağırlık katsayısı-2 (wc2) = $0,3,0,5,0,7$ için 10 farklı çalışmadan seçilen en iyi performansin yinelenen tipik maliyet ve fonksiyon hesaplama sayısı (FEN) varyasyonlarını sırası ile ateşböceği, diferansiyel evrim ve guguk kuşu algoritmaları için göstermektedir. Ayıca, Şekil 5, Şekil 6 ve Şekil 7 'deki maliyet ve FEN varyasyonları sayısal tablo olarak Tablo 5, Tablo 6 ve Tablo 7'de verilmiştir.

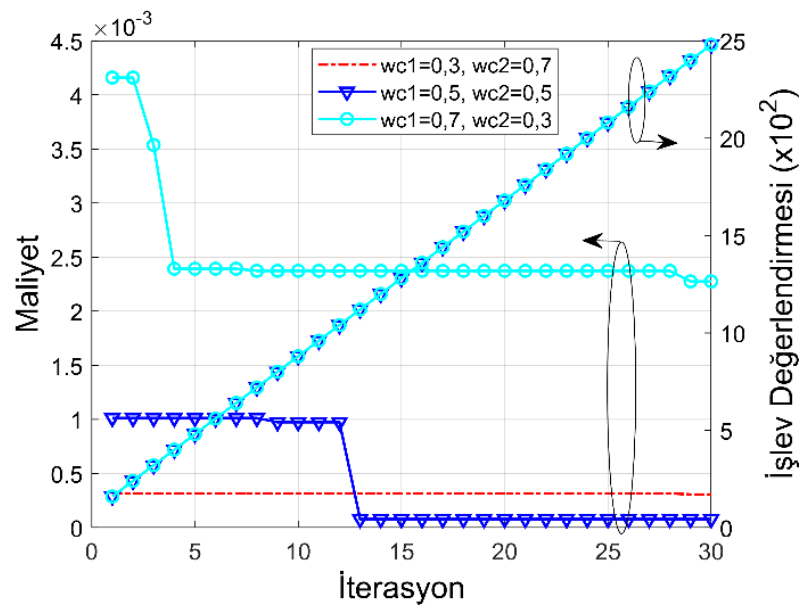

Şekil 5. Ateşböceği algoritması için ă̆ırlık katsayılarına göre 10 farklı koşudan seçilen optimizasyonun en iyi performansinın yinelemeli tipik maliyet ve FEN varyasyonlart (popülasyon $(N)=80)$

Figure 5. Typical cost and FEN variations with iteration of the best performance of optimization selected from 10 different runs by weight coefficients for the firefly algorithm (population $(N)=80)$ 


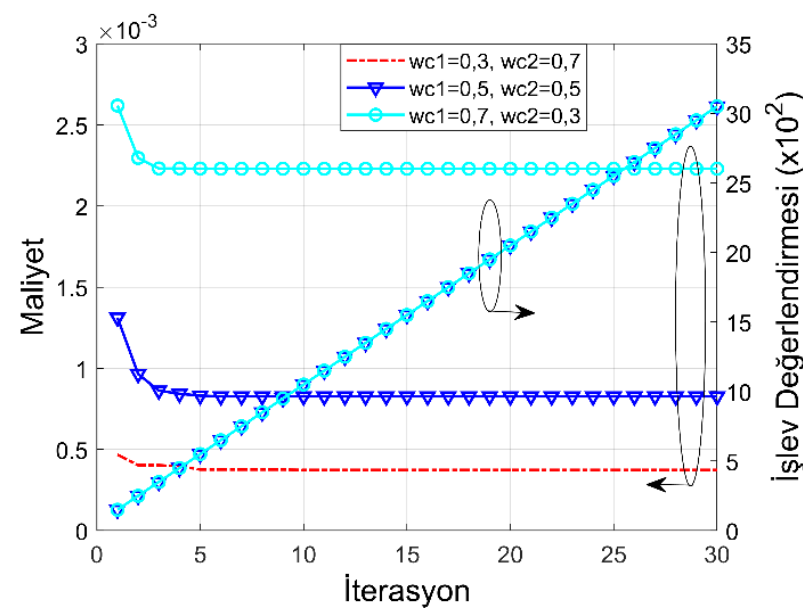

Şekil 6. Diferansiyel evrim algoritmast için ă̆ırlı katsayılarına göre 10 farklı koşudan seçilen optimizasyonun en iyi performansının yinelemeli tipik maliyet ve FEN varyasyonlar (popülasyon $(N)=50)$

Figure 6. Typical cost and FEN variations with iteration of the best performance of optimization selected from 10 different runs by weight coefficients for the differential evolution algorithm (population $(N)=50$ )

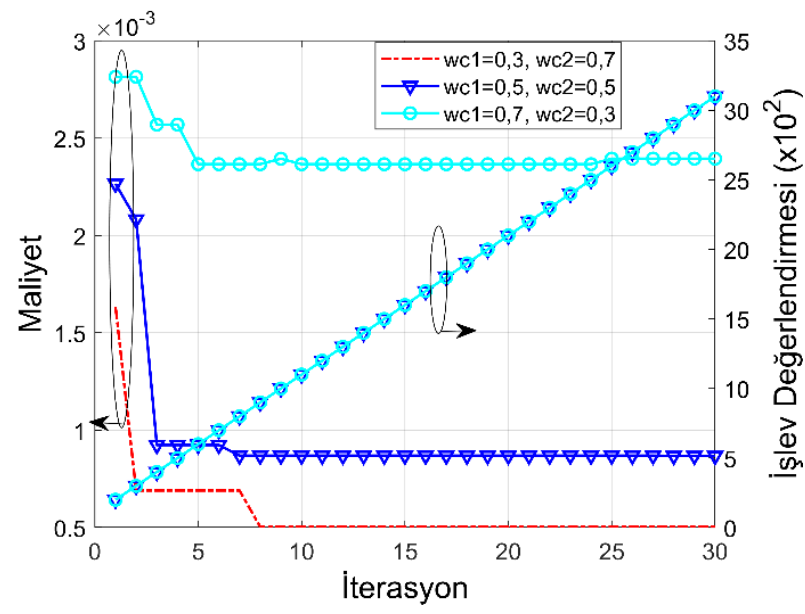

Şekil 7. Guguk kuşu arama algoritması için ă̆ırlı katsayılarına göre 10 farklı koşudan seçilen optimizasyonun en iyi performansinin yinelemeli tipik maliyet ve FEN varyasyonlar (popülasyon $(N)=50)$

Figure 7. Typical cost and FEN variations with iteration of the best performance of optimization selected from 10 different runs by weight coefficients for the cuckoo search algorithm $($ population $(N)=50)$
Tablo 5. Ateşböceği algoritması için ağırlık katsayllarına göre optimizasyonun performans değerlendirmeleri (popülasyon $(N)=80$ )

Table 5. Performance evaluations of optimization by weight coefficients for the firefly algorithm (population $(N)=80$ )

\begin{tabular}{cccccc}
\hline wc1 & wc2 & & Minimum & Maksimum & Ortalama \\
\hline \multirow{2}{*}{0,3} & 0,7 & Maliyet & $3,07 \mathrm{E}-04$ & 0,000316109 & $3,16 \mathrm{E}-04$ \\
& & FEN & 2400 & 160 & 2480 \\
0,5 & 0,5 & Maliyet & $7,89304 \mathrm{E}-05$ & $1,01 \mathrm{E}-03$ & $4,47 \mathrm{E}-04$ \\
& & FEN & 1120 & 160 & 2480 \\
0,7 & 0,3 & Maliyet & 0,002276241 & $4,16 \mathrm{E}-03$ & $2,53 \mathrm{E}-03$ \\
& & FEN & 2400 & 160 & 2480 \\
\hline
\end{tabular}

Tablo 6. Diferansiyel evrim algoritması için ağırlık katsayılarına göre optimizasyonun performans değerlendirmeleri (popülasyon $(N)=$ 50)

Table 6. Performance evaluations of optimization by weight coefficients for the differential evolution algorithm (population $(N)=50$ )

\begin{tabular}{cccccc}
\hline wc1 & wc2 & & Minimum & Maksimum & Ortalama \\
\hline \multirow{2}{*}{0,3} & 0,7 & Maliyet & $3,74 \mathrm{E}-04$ & 0,000467979 & $3,80 \mathrm{E}-04$ \\
& & FEN & 1050 & 150 & 3050 \\
0,5 & 0,5 & Maliyet & 0,000826929 & $1,31 \mathrm{E}-03$ & $8,50 \mathrm{E}-04$ \\
& & FEN & 2350 & 150 & 3050 \\
0,7 & 0,3 & Maliyet & 0,002229796 & $2,62 \mathrm{E}-03$ & $2,25 \mathrm{E}-03$ \\
& & FEN & 2450 & 150 & 3050 \\
\hline
\end{tabular}


Tablo 7. Guguk kuşu arama algoritması için ăgırlık katsayılarına göre optimizasyonun performans değerlendirmeleri (popülasyon $(N)=$ 50)

Table 7. Performance evaluations of optimization by weight coefficients for the cuckoo search algorithm (population $(N)=50$ )

\begin{tabular}{cccccc}
\hline wc1 & wc2 & & Minimum & Maksimum & Ortalama \\
\hline \multirow{2}{*}{0,3} & 0,7 & Maliyet & $5,04 \mathrm{E}-04$ & 0,001635864 & $9,71 \mathrm{E}-04$ \\
& & FEN & 900 & 200 & 3100 \\
0,5 & 0,5 & Maliyet & 0,000868378 & $2,27 \mathrm{E}-03$ & $9,64 \mathrm{E}-04$ \\
& & FEN & 2900 & 200 & 3100 \\
0,7 & 0,3 & Maliyet & 0,002366233 & $7,03 \mathrm{E}-03$ & $3,46 \mathrm{E}-03$ \\
& & FEN & 600 & 200 & 3100 \\
\hline
\end{tabular}

\section{Yuvarlak Papyon Anten için Geri Dönüş Kaybı}

Şekil 8, Şekil 9 ve Şekil 10 popülasyon $(\mathrm{N})=30$, 50 ve 80 değerleri için en iyi performansa sahip üç antenin geri dönüş kaybını $\left(\mathrm{S}_{11}\right)$ 4-6 GHz arasında tipik büyüklük ve frekans değişimlerini sırası ile ateşböceği, diferansiyel evrim ve guguk kuşu arama algoritmaları için göstermektedir. Bunun yanı sıra, Şekil 8, Şekil 9 ve Şekil 10'daki büyüklük ve frekanstaki değişimler sayısal olarak Tablo 8, Tablo 9 ve Tablo 10 'da verilmiştir. Sonuçlardan görüleceği üzere popülasyon değerini arttırmak her zaman optimizasyonun yükünü attırırken her zaman en düşük maliyetli sonucu vermediği görülmektedir.

Şekil 11, Şekil 12 ve Şekil 13'de diferansiyel evrim ve guguk kuşu arama algoritmaları için popülasyon $(\mathrm{N})=50$ ve ateşböceği algoritması için ise popülasyon $(\mathrm{N})=80$ parametrelerine ek olarak ağırlık katsayısı-1 (wc1) ve ağırlık katsayıs1-2 $($ wc2 $)=0,3,0,5,0,7$ için en iyi üç performanslı antenin geri dönüş kaybını $\left(S_{11}\right)$ 4-6 $\mathrm{GHz}$ arasında tipik büyüklük ve frekans değişimlerini sırası ile ateşböceği, diferansiyel evrim ve guguk kuşu arama algoritmaları için göstermektedir. Ek olarak, Şekil 11, Şekil 12 ve Şekil 13'deki büyüklük ve frekanstaki değişimler sayısal olarak Tablo 8, Tablo 9 ve Tablo 10 'da verilmiştir. Ağırlık katsayısının (wc1) artmasının geri dönüş kaybı sonuçlarına yansıdığı görülmektedir. Ayrıca tüm şekillerdeki ortalama sonuçlara göre antenlerin bant genişliklerinin, 4,5-5,5 GHz arasında olduğu söylenebilir.

Bununla birlikte, önerilen anten tasarımı ateşböceği algoritması ile tasarlanan popülasyon $(\mathrm{N})=80$ ve ağırlık katsayısı-1 $(\mathrm{wc} 1)=0,7$ ve ağırlık katsayısı-2 (wc2) = 0,3 için $5 \mathrm{GHz}$ rezonans frekans noktasında geri dönüş kaybı $\left(\mathrm{S}_{11}\right)-56,31 \mathrm{~dB}$ iken sirasiyla $1,96 \mathrm{~dB}, 1,95 \mathrm{~dB}$ ve $1,94 \mathrm{~dB}$ olmak üzere sirasi ile 30,60 ve 90 derecelerde kazanç değerlerine sahiptir. Ayrıca önerilen anten, belirtilen frekans bandı için \%60 ve üzeri bir verime sahiptir.

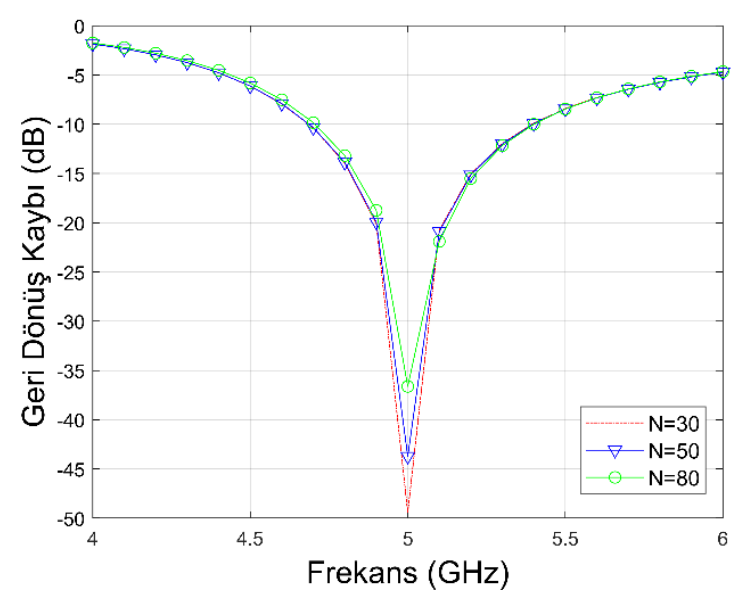

Şekil 8. Ateşböcĕ̆i algoritması için farklı popülasyon değerleri arasından seçilen en iyi performansa göre antenin geri dönüş kaybı

Figure 8. Return loss of the antenna according to the best performance selected among different population values for the firefly algorithm 


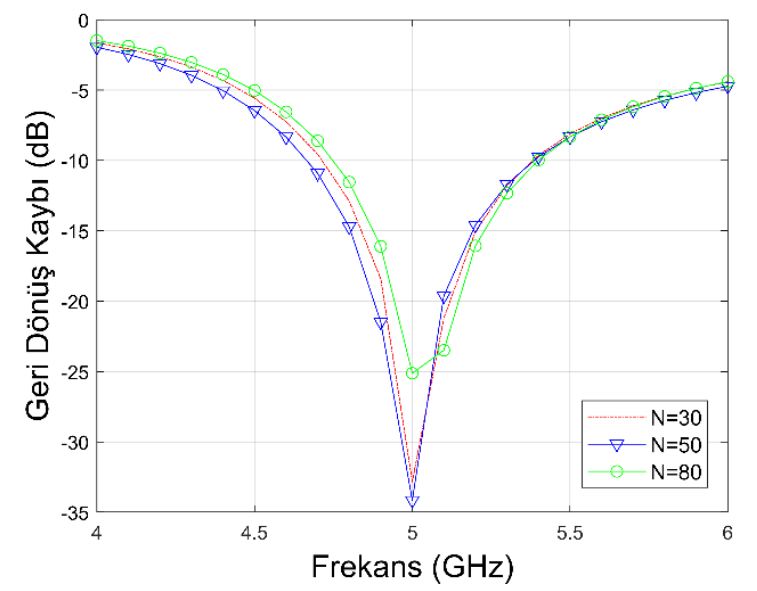

Şekil 9. Diferansiyel evrim algoritması için farklı popülasyon değerleri arasından seçilen en iyi performansa göre antenin geri dönüş kaybı

Figure 9. Return loss of the antenna according to the best performance selected among different population values for the differential evolution algorithm

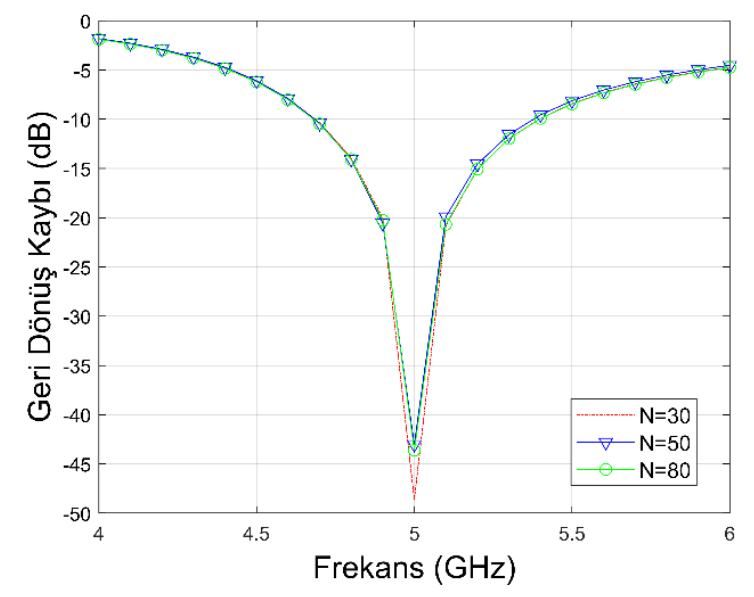

Şekil 10. Guguk kuşu arama algoritması için farkl popülasyon değerleri arasından seçilen en iyi performansa göre antenin geri dönüş kaybı

Figure 10. Return loss of the antenna according to the best performance selected among different population values for the cuckoo search algorithm

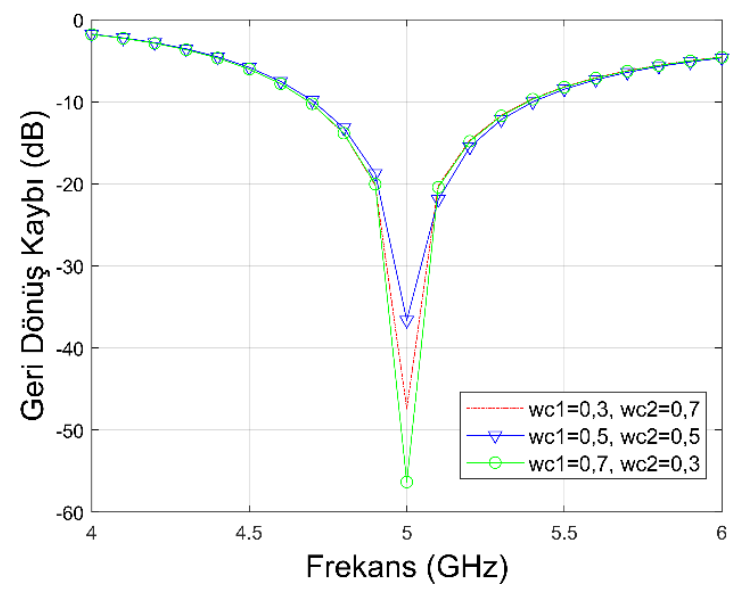

Şekil 11. Ateşböceği algoritması için farklı ă̆ırlık katsayı değerleri arasından seçilen en iyi performansa göre antenin geri dönüş kaybı (popülasyon $(N)=80$ )

Figure 11. Return loss of the antenna according to the best performance selected among different weight coefficient values for the firefly algorithm (population $(N)=80)$

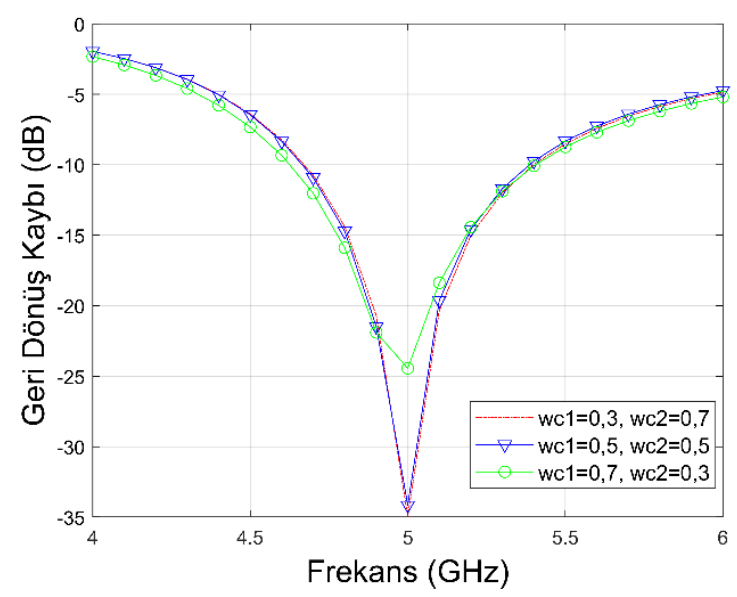

Şekil 12. Diferansiyel evrim algoritması için farkl ă̆ırlık katsayı değerleri arasından seçilen en iyi performansa göre antenin geri dönüs kaybı $($ popülasyon $(N)=50)$

Figure 12. Return loss of the antenna according to the best performance selected among different weight coefficient values for the differential evolution algorithm (population $(N)=50$ ) 


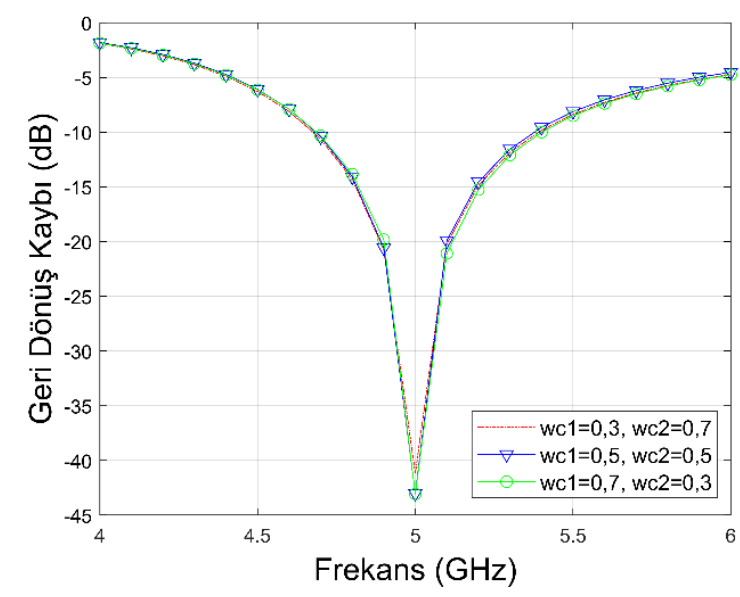

Şekil 13. Guguk kuşu arama algoritması için farklı ağırlık katsayı değerleri arasından seçilen en iyi performansa göre antenin geri dönüss kaybı $($ popülasyon $(N)=50)$

Figure 13. Return loss of the antenna according to the best performance selected among different weight coefficient values for the cuckoo search algorithm (population $(N)=50$ )

Tablo 8. Şekil 8 ve Şekil 11'deki geri dönüş kaybı $\left(S_{11}\right)$ değerlerinin sayısal formu

Table 8. Numerical form of return loss $\left(S_{11}\right)$ values in Figure 8 and Figure 11

\begin{tabular}{cccc}
\hline wc1 & wc2 & $\begin{array}{c}\text { Popülasyon } \\
(\mathrm{N})\end{array}$ & $\begin{array}{c}\text { Geri Dönüş Kayb1 } \\
\left(\mathrm{S}_{11}\right)\end{array}$ \\
\hline 0,5 & 0,5 & 30 & $-49,4$ \\
0,5 & 0,5 & 50 & $-43,71$ \\
0,5 & 0,5 & 80 & $-36,61$ \\
0,3 & 0,7 & 80 & $-47,4$ \\
0,7 & 0,3 & 80 & $-56,31$ \\
\hline
\end{tabular}

Tablo 9. Şekil 9 ve Şekil 12'daki geri dönüss kaybı $\left(S_{11}\right)$ değerlerinin sayısal formu

Table 9. Numerical form of return loss $\left(S_{11}\right)$ values in Figure 9 and Figure 12

\begin{tabular}{cccc}
\hline wc1 & wc2 & $\begin{array}{c}\text { Popülasyon } \\
(\mathrm{N})\end{array}$ & $\begin{array}{c}\text { Geri Dönüş Kayb1 } \\
\left(\mathrm{S}_{11}\right)\end{array}$ \\
\hline 0,5 & 0,5 & 30 & $-32,83$ \\
0,5 & 0,5 & 50 & $-34,15$ \\
0,5 & 0,5 & 80 & $-25,12$ \\
0,3 & 0,7 & 50 & $-34,94$ \\
0,7 & 0,3 & 50 & $-24,43$ \\
\hline
\end{tabular}

Tablo 10. Şekil 10 ve Şekil 13'deki geri dönüş kaybu $\left(S_{11}\right)$ değerlerinin sayısal formu

Table 10. Numerical form of return loss $\left(S_{11}\right)$ values in Figure 10 and Figure 13

\begin{tabular}{cccc}
\hline wc1 & wc2 & $\begin{array}{c}\text { Popülasyon } \\
(\mathrm{N})\end{array}$ & $\begin{array}{c}\text { Geri Dönüş Kayb1 } \\
\left(\mathrm{S}_{11}\right)\end{array}$ \\
\hline 0,5 & 0,5 & 30 & $-48,62$ \\
0,5 & 0,5 & 50 & $-43,01$ \\
0,5 & 0,5 & 80 & $-43,61$ \\
0,3 & 0,7 & 50 & $-41,18$ \\
0,7 & 0,3 & 50 & $-43,06$ \\
\hline
\end{tabular}

\section{Sonuçlar}

Bu çalışmada, kablosuz yerel ağları (WLAN) ve iletişim sistemlerinde çalışabilen tek bant yuvarlak papyon anten tasarımları, rekabetçi evrimsel algoritmalar ile bir optimizasyon problemi olarak formüle edilmiş ve antenlerin geometrik tasarım parametrelerine göre çözümleri maliyet fonksiyonu olarak ifade edilmiştir. Ateşböceği (FF), diferansiyel evrim (DE) ve guguk kuşu arama (CS) algoritmaları; moment yöntemi (MoM) tekniği kullanılarak elde edilen maliyet fonksiyonu kullanılarak, optimum tasarım değerlerini elde etmek üzere başarıyla uygulanmıştır. $\mathrm{Bu}$ sonuçlarda ateş böceği, diferansiyel evrim ve guguk kuşu algoritmaları için en iyi geri dönüş kaybı $\left(S_{11}\right)$ değerleri sırası ile $-56,31 \mathrm{~dB},-34,94 \mathrm{~dB}$ ve $-48,62 \mathrm{~dB}$ olarak bulunmuştur. Ayrıca yönlendiricilik kazanç değerleri sırası ile $1,94 \mathrm{~dB}, 1,93 \mathrm{~dB}$ ve $1,94 \mathrm{~dB}$ hesaplanmıştır. $\mathrm{Bu}$ üç rekabetçi algoritma arasından, ateşböceği ile optimize edilen sonuç en başarılı olarak seçildi. Elde edilen simülasyon sonuçları, grafiksel şekil olarak ve sayısal form olarak bakıldığında önerilen üç rekabetçi tasarım optimizasyon yöntemleriyle başarılı sonuçlar elde edildiği söylenebilir. $\mathrm{Bu}$ optimizasyon teknikleri performans değerlendirmeleri açısından bir dipol antenin optimal çözümlerini oluşturmak için etkili algoritmalardır. Ek olarak, önerilen tasarım optimizasyon süreçleri tek bant yuvarlak papyon anten tasarımı ile sinırlı kalmayıp, aynı zamanda tasarım optimizasyon hedefleri değiştirilerek başka mikrodalga devre tasarımı problemleri için de başarıyla uygulanabilir. 


\section{Kaynaklar}

[1] Su, C. M., Chen, W. S., Wong, K. L., (2003). Compact dual-band metal-plate antenna for 2.4/5.2GHz WLAN operation, Microw. Opt. Technol. Lett., 38, 113-115.

[2] Gao, X., Zhong, H., Zhang, Z., Feng, Z., Iskander, M. F., (2010). Low-profile planar tripolarization antenna for WLAN communications, IEEE Antennas Wireless Propag. Lett., 9, 83-86.

[3] Wong, K. L., Chou, L. C., Su, C. M., (2005). Dualband flat-plate antenna with a shorted parasitic element for laptop applications, IEEE Trans. Antennas Propag., 53, 1, 539-544.

[4] Chang, T. N., Jiang, J.-H., (2009), Meandered Tshaped monopole antenna, IEEE Trans. Antennas Propag., 57, 12, 3976-3978.

[5] Nakano, H., Sato, Y., Mimaki, H., Yamauchi, J., (2005). "An inverted FL antenna for dual-frequency operation," IEEE Trans. Antennas Propag., 53, 8, 2417-2421.

[6] Li, R., Pan, B., Laskar, J., Tentzeris, M. M., (2008). A novel low-profile broadband dual-frequency planar antenna for wireless handsets, IEEE Trans. Antennas Propag., 56, 4, 1155-1162.

[7] Jan, J. Y., Tseng, L. C., (2004). Small planar monopole antenna with a shorted parasitic inverted-L wire for wireless communications in the 2.4-, 5.2-, and 5.8-GHz bands, IEEE Trans. Antennas Propag., 52, 7, 1903-1905.

[8] Liu, W. C., Wu, C. M., Dai, Y., (2011). Design of triple-frequency microstrip- fed monopole antenna using defected ground structure, IEEE Trans. Antennas Propag., 59, 7, 2457-2463.

[9] Minasian, A. A., Bird, T. S., Atai, J., (2011). Particle swarm antennas for wireless communication systems," in Proc. EuCAP, Rome, Italy, 897-899.

[10] Minasian, A. A., Bird, T. S., (2012). Complementary particle swarm antennas for next generation wireless communication systems," in Proc. ISWCS, Paris, France, 895-898.

[11] Balanis, C. A., (2005). Antenna Theory: Analysis and Design, 3rd ed. Hoboken, NJ, USA: Wiley.

[12] Fisher, L., (2009). The Perfect Swarm. New York, NY, USA: Basic Books.

[13] Robinson, J., Sinton, S., Rahmat-Samii, Y., (2002). Particle swarm, genetic algorithm, and their hybrids: optimization of a profiled corrugated horn antenna, in Proc. IEEE Int. Symp. Antennas Propagation, San Antonio, 1, 314-317.
[14] Boeringer, D., Werner, D., (2004). Particle swarm optimization versus genetic algorithms for phased array synthesis, IEEE Trans. Antennas Propag., 52, 3, 771-779.

[15] Yang, F., Zhang, X-X., Ye, X., Rahmat-Samii, Y., (2001). Wide-band E-shaped patch antennas for wireless communications, IEEE Trans. Antennas Propag., 49, 7, 1094-1100.

[16] Kim, T-W., Choi, K., Hwang, H-Y., (2015). Small bowtie monopole UWB antenna, Loughborough Antennas \& Propagation Conference (LAPC).

[17] Saghlatoon, H., Honari, M. M., Mirzavand, R., Mousavi, P., Kumar, A., La, T. G., Chung, H-J., (2017). A novel investigation on printed stretchable WLAN antennas, IEEE International Symposium on Antennas and Propagation \& USNC/URSI National Radio Science Meeting.

[18] Hamid, A-K., Obaid, W., (2017). Penta-frequency CPW bow-tie aperture antenna for mobile communications, 7th International Conference on Modeling, Simulation, and Applied Optimization (ICMSAO).

[19] Mulchandani, J. D., Gupta, D., Sharma, S. K., Mishra, N., Chaudhary, R. K., (2016). Narrow-band electrically small metamaterial-inspired antenna with bowtie-shaped stub for WLAN, 11th International Conference on Industrial and Information Systems (ICIIS).

[20] Mansoul, A., (2017). Reconfigurable multiband bowtie antenna for WiFi, WiMax, and WLAN applications, IEEE International Symposium on Antennas and Propagation \& USNC/URSI National Radio Science Meeting.

[21] Zhang, Q-L., Si, L-M., Wu, Y-M., Liu, Y., Lv, X., (2014). Design of a coplanar bowtie antenna for WLAN and WiMAX application, Proceedings of 2014 3rd Asia-Pacific Conference on Antennas and Propagation.

[22] Belen, M. A., Alıcı, M., Çor, A., Güneş, F., (2014). Performance characterization of a microwave transistor using firefly Algorithm' Symposium of electrical- electronics and computer engineering ELECO, 27, 29, 491-493.

[23] Yang, X.S., (2009). Firefly algorithms for multimodal optimization', Stochastic algorithms: Foundations and applications, SAGA, Lecture notes in computer sciences, 5792, 169-178.

[24] Yang, X. S., Deb, S., (2010). Engineering optimization by cuckoo search', Int. J. Mathematical modelling and numerical optimization, 1, 4, 330-343.

[25] Wang, F., He, X. S., Wang, Y., Yang, S., (2012). Markov model and convergence analysis based on cuckoo search algorithm', Jisuanji Gongcheng/ Computer Engineering, 38, 11, 180-185. 
[26] Yang, X. S, Deb, S., (2009). Cuckoo Search via Levy Flights', Proc. of world congress on nature \& biologically inspired computing, 210-214.

[27] Güneş, F., Karataev, T., Demirel, S., (2016). Composite right/left-handed transmission lines in use for ultrawideband matching of front-end amplifiers with modified cuckoo search optimization', International Journal of Numerical Modelling: Electronic networks, devices and Fields, DOI: 10.1002/jnm.21441.

[28] Storn, R., Price, K., (1997). Differential evolution - a simple and efficient heuristic for global optimization over continuous spaces', Journal of global optimization, 11, 4, 341-359.
[29] Das, S., Abraham, A. Chacraborty, U.K., Konar, A., (2009). Differential evolution using a neighborhoodbased mutation operator', IEEE Transactions on evolutionary computation, 13, 3, 526-553.

[30] Das, S., Suganthan, P.N., (2011). Differential Evolution: A Survey of the State-of-the-Art', IEEE Transactions on Evolutionary Computation, 15, 1, 431.

[31] Yang, X-S., (2013). Multiobjective Firefly Algorithm for Continuous Optimization, Engineering with Computers, Engineering with Computers, 29, 2, 175184.

[32] Johnson, J.M., Rahmat-Samii, Y., (1999). Genetic algorithms and method of moments (GA/MOM) for the design of integrated antennas, IEEE Transactions on Antennas and Propagation, 47, 10, 1606-1614. 